\title{
Digitalización de una escuela de turismo para garantizar su viabilidad tras la Covid-19
}

\author{
Diego Santos González, Raúl Travé Molero, \\ Jennifer Rodríguez Martín, \\ dsantos@ostelea.com,rtrave@ostelea.com \\ jrodriguezma@ostela.com \\ Ostelea Tourism Management School
}

Resumen. Con la llegada de la pandemia (marzo de 2020), el departamento académico del campus de Madrid de una escuela universitaria privada de turismo se vio en la necesidad de digitalizarse en tiempo récord, no solo para afrontar una nueva realidad laboral desde el hogar, sino también para virar desde un modelo de negocio basado en la presencialidad hacia una nueva realidad en la que ya no se podían ofrecer los productos (másteres) de la misma manera en que se habia becho antes de la pandemia.

La viabilidad de la escuela está directamente relacionada con el número de matriculas de cada convocatoria, por lo que se debian afrontar dos problemas. En primer lugar, ofrecer una solución al alumnado que estaba cursando y vio sus estudios interrumpidos por la llegada de la pandemia, y, en segundo lugar, babia que trabajar en prospectiva, garantizando que las matrículas de las siguientes convocatorias no se cancelasen. El presente artículo revisa críticamente las medidas adoptadas desde el departamento académico para garantizar la viabilidad y el futuro de la escuela en un escenario marcado por las dificultades de movilidad y las restricciones sanitarias.

Palabras clave: Digitalización; escuelas de negocios; covid-19; metodología online; metodología hibrida; investigación-acción participativa. 
Digitalization in a tourism SChOol to ENSURE its Viability AFTer Covid-I9

Abstract. With the arrival of the pandemic (March 2020), the academic department of a private tourism school in Madrid realised that it had to digitize in record time not only to face a new situation in which people had to work from home, but also to shift from a business model based on physical presence to one in which master's degrees could no longer be offered in the same way as they had been done before the pandemic. The viability of the school is directly related to the number of students who register for each academic year, so two problems had to be faced. In the first place, the school needed to offer a solution to the students who were already studying and had had their studies interrupted by the arrival of the pandemic and, in the second place, the school needed to work prospectively to ensure that registrations of new students for the subsequent academic years were not cancelled. This article critically reviews the measures adopted by the academic department to guarantee the viability and future of the school in a scenario marked by mobility difficulties and health restrictions.

Keywords: Digitization; Business schools; Covid-19; Online methodology; Hybrid methodology; Participatory-Action Research 


\section{Introducción}

En este artículo vamos a exponer los principales aprendizajes de nuestra experiencia como responsables del departamento académico de una escuela universitaria de turismo en la doble adaptación que, en pocos meses, se hizo a la docencia en línea, primero, y a la docencia híbrida, después, para poder garantizar la prestación de servicios durante la pandemia de covid-19 que estamos viviendo desde el año 2020.

Tras unos primeros días de incertidumbre en el mes de marzo de 2020, en los que parecía que todo apuntaba a la necesidad de una mera adaptación del calendario académico, pronto se descubrió que la vuelta a las clases presenciales no era viable en el corto plazo. El modelo de negocio había mutado y la escuela tuvo la necesidad operativa de transformar la docencia presencial en virtual, primero, $y$ en semipresencial, una vez que se redujeron las restricciones de movilidad.

Gracias a que otra división de la escuela también tiene productos completamente digitales, disponíamos de una plataforma online en la que continuar prestando servicios. A priori, teníamos la capacidad operativa de convertirnos en un centro de docencia online, sin embargo, el proceso no fue tan sencillo.

La digitalización estuvo llena de problemas desde el principio. La necesidad de solucionarlos y protocolizarlos nos forzó a construir sobre la marcha una suerte de investigación-acción participativa (IAP) en la que han participado más de 200 profesores y cerca de mil estudiantes de los dos campus de la escuela. No obstante, aquí nos centraremos en el caso de Madrid, con 158 alumnos repartidos en siete programas, 72 profesores y 6 directores de programa.

Tras un sondeo cualitativo usando como informantes a delegados de clase $y$ directores de programa, descubrimos que, a pesar del uso habitual de una plataforma digital de enseñanza, el principal obstáculo, aunque más marcado en profesores que en estudiantes, era el desconocimiento de la mayoría de las herramientas digitales disponibles, así como la ausencia de conocimientos técnicos básicos para organizar una clase en línea.

Solo una vez superados estos problemas, que nos situaban en la mera transposición de la docencia tradicional, pudieron empezar a plantearse innovaciones en el plano metodológico que ayudasen a ofrecer el mejor servicio posible, cuestión capital en la estrategia empresarial. El posterior paso a la docencia híbrida ejecutado en el mes de octubre de 2020 supuso, en buena medida, una vuelta a empezar en este proceso, pues exige combinar los mundos presencial y telemático sin olvidar a los estudiantes que por razones horarias ${ }^{1}$ deben ver en diferido las clases sin sentirse ajenos a estas y sin perder su derecho a la evaluación continua.

1 Estudiantes extranjeros que no han podido desplazarse a España a causa de la pandemia de covid-19. 
El perfil del alumnado es principalmente internacional, tanto en los programas en español como en los que se imparten en inglés, por lo que, además de todas las circunstancias relacionadas con la docencia, la incertidumbre inicial, los cierres de los espacios aéreos y los problemas burocráticos con la concesión y extensión de visados no hacían más que complicar la situación tanto a los alumnos que estaban matriculados durante el curso 2019/2020 como a los posibles alumnos prospectivos, algunos de los cuales ya habían realizado sus matrículas para el curso 2020/2021 y esperaban saber qué metodología docente se ofrecería como alternativa a la tradicional, basada en la presencialidad.

Fuimos, por tanto, los encargados de, en pocos días, transformar las clases presenciales en virtuales y trasladar las actividades planificadas para realizarse presencialmente durante los meses finales del curso 2019/2020 a un entorno virtual. Además, diseñamos un plan con el que, tras el confinamiento, adaptar nuestra escuela a los protocolos covid-19 y poder continuar ofreciendo nuestros másteres de manera presencial, telemática e híbrida. Estas dos últimas, tras identificar durante los meses de verano que un porcentaje bastante elevado del alumnado que debía comenzar en octubre quería cursar presencialmente, pero desconocía cuándo podría incorporarse por las limitaciones de movilidad. Esto suponía un problema logístico que debíamos superar.

La evaluación de los resultados de las encuestas de satisfacción de los estudiantes indica que, a pesar de las dificultades, la docencia en línea ha conseguido cubrir los contenidos de los currículos. No obstante, la metodología todavía debe mejorarse y sistematizarse para alcanzar las expectativas de los estudiantes. La información obtenida a través de las encuestas de satisfacción resultó, además, de gran utilidad en el diseño de la metodología docente híbrida.

Gracias a las conclusiones de este esfuerzo entendemos que podemos establecer una serie de buenas prácticas para la digitalización de una escuela de formación universitaria que pueden ayudar a otras instituciones que se encuentren en una situación similar.

\section{Objetivos}

A lo largo de este artículo analizaremos las diferentes situaciones y soluciones adoptadas, a partir de una metodología IAP, en el ámbito de una escuela universitaria con el objetivo general de transformar la docencia presencial en docencia totalmente telemática en un primer momento, el segundo cuatrimestre del curso 2019/2020, y en docencia híbrida para todo el curso 2020/2021, sin sacrificar la calidad educativa. Por tanto, en todo momento, la pregunta que ha guiado la IAP ha sido qué problemas o dificultades encuentran profesores y alumnos en el pro- 
ceso de digitalización. Siguiendo el espíritu de la metodología IAP las respuestas han sido una construcción colectiva que se ha ido reformulando según avanzaba la investigación.

Además, expondremos los pasos y resultados más relevantes:

- La identificación de los problemas a los que se enfrentó el claustro para adaptarse a la formación online durante el confinamiento del curso 2019/2020.

- El análisis de las acciones y adaptaciones llevadas a cabo por el centro para transformar la docencia presencial en docencia telemática en el curso $2019 / 2020$.

- El plan de acción adoptado por el centro para poder ofertar docencia presencial en el curso 2020/2021.

- Los problemas a los que se enfrenta el claustro ante la docencia híbrida en el curso 2020/2021.

- La medición del grado de satisfacción del alumnado del curso 2019/2020 en relación con las soluciones adoptadas por la escuela tras el confinamiento.

- Los resultados preliminares de satisfacción del alumnado del curso 2020/2021 en relación con la docencia híbrida.

\section{Fundamentación teórica}

Llevamos varias décadas en las que gran parte del discurso sobre la innovación metodológica de la enseñanza se ha centrado en la importancia que tienen las TIC. Se trata, por tanto, de una cuestión que ha sido ampliamente abordada, de manera teórica y práctica, por multitud de autores.

El giro constructivista en la relación entre docentes y discentes (Prawat, 1992) puso las bases para un modelo de docencia superador de la perspectiva objetivista clásica, con un peso creciente del papel activo y colaborativo de los estudiantes. Este cambio en los modelos docentes se vio reforzado desde mediados de los años noventa del siglo xx con la popularización de internet y de los cursos en línea que pronto empezaron a surgir y difundirse, también en el ámbito universitario (Azcorra et alii, 2001). No obstante, uno de los males endémicos de la enseñanza telemática ha sido la «trasposición directa de estrategias y prácticas previas, anteriores» (Adell, 2004: 57). En este sentido, Harmon y Jones (1999: 28-29) ya señalaban la existencia de varios niveles de uso de las herramientas digitales en educación. Estos niveles van del 0, en el que no se usan estas herramientas, o solo para transmitir información institucional, hasta el 5, en el que se 
crea una comunidad virtual de aprendizaje constructivista alejada de las formas tradicionales de educación. Entre los dos extremos de este continuo podemos encontrar el nivel 1, en el que se proporciona a través de una web información académica básica (calendarios, tutorías, etc.); el nivel 2, en el que se ponen a disposición de los estudiantes algunos materiales digitales (apuntes, presentaciones, etc.); el nivel 3, en el que la mayoría de los materiales del curso se obtienen accediendo a una web, y el nivel 4, que sería un modelo de enseñanza-aprendizaje mixto, con encuentros presenciales e interacción en línea.

Obras ya clásicas, como e-Moderating: the key to teaching and learning online, de Gylly Salmon (2000), presentan el papel del docente en la enseñanza telemática como el de un moderador digital cuya misión es promover la comunicación y la interacción en el proceso de construcción de conocimientos y competencias. Salmon divide este proceso en cinco etapas: 1) acceso al entorno digital y motivación de los estudiantes para que lo conozcan y usen; 2) socialización en línea; 3) intercambio de información y apoyo en el uso de materiales de enseñanza y realización de tareas; 4) construcción de los conocimientos, y 5) desarrollo y ampliación de los conocimientos.

En el proceso de moderación y construcción conjunta de los conocimientos y habilidades que deben alcanzar los estudiantes juegan un papel central los materiales específicos que se usarán en el entorno digital (Jolliffe, Ritter y Stevens, 2001; Garrison y Anderson, 2005). Del mismo modo, la naturaleza de este tipo de enseñanza enfrenta al docente a una realidad social, cultural y geográfica especialmente diversa y a unas necesidades de evaluación y medición de resultados significativamente diferentes a la enseñanza tradicional (Pallof y Pratt, 2001).

Sin embargo, los problemas asociados a este modelo también empezaron a ser patentes en los primeros años de implementación, fundamentalmente la falta de comunicación entre profesores y estudiantes y entre los propios estudiantes (Schell, 2001). Diversos autores han señalado la importancia de la comunidad de aprendizaje: «[... ] la comunidad es el vehículo a través del cual ocurre el aprendizaje online [...]. Sin el soporte y participación de una comunidad de aprendizaje, no existe el curso online» (Palloff y Pratt, 1999: 29). En este sentido, algunos autores han señalado el error del tecnocentrismo (Cabero, 2006: 4), es decir, se ha confiado en exceso en las virtudes intrínsecas de la tecnología, minusvalorando la importancia de la didáctica y la pedagogía. Para estos autores, la formación telemática debería promover formas de aprendizaje cooperativas y la creación de comunidades virtuales capaces de superar problemas habituales en este tipo de formación, como «el sentido de aislamiento y de soledad de los estudiantes» (Cabero, 2006: 8). 
Actualmente ha tomado fuerza la idea de incluir herramientas digitales en las dinámicas de las clases presenciales, especialmente los teléfonos inteligentes (Sung et alii, 2016), para crear sistemas mixtos de formación y de forjar comunidades cooperativas dentro de ecosistemas tecnológicos de enseñanza (GarcíaPeñalvo, 2018).

A pesar de la experiencia y los conocimientos acumulados en el ámbito de la educación telemática, el confinamiento que experimentamos en España (y en buena parte del mundo) en 2020 por culpa de la covid-19 mostró que Administraciones, docentes y alumnos no estábamos suficientemente preparados para la utilización de estas herramientas tecnológicas enfocadas a la docencia no presencial.

\section{Metodología}

En la presente investigación se ha implementado una estrategia metodológica basada en la investigación-acción participativa, siguiendo las reflexiones de Miguel Martínez (2009) sobre la vertiente educativa de esta, un tipo de investigación que tiende a confundirse con la vida normal del aula (Martínez, 2009: 243). Este tipo de investigación permite investigar para comprender los problemas en el aula (digital en nuestro caso) y para solucionarlos. En el proceso, además, los participantes (coinvestigadores) mejoran sus competencias a través de un proceso de retroalimentación constante y su participación activa en la definición del problema y el análisis de los resultados (Martínez, 2009: 239-240). Esta perspectiva nos permite partir de la especificidad de la situación concreta en la aplicación de leyes generales (Martínez, 2009: 241).

Al igual que autores como Paulo Freire (1974), Hilda Taba (1957) o John Elliot (1990), consideramos que esta metodología es realmente interesante para analizar problemáticas docentes, y, en nuestro caso, entendíamos que podía ser extremadamente útil para buscar soluciones en un corto período de tiempo.

$\mathrm{Al}$ amparo de esta metodología, la investigación ha sido modulada y secuenciada en diferentes fases. Tal y como asegura Ana Mercedes Colmenares, existen diferentes denominaciones para las diferentes fases sin que esto suponga ningún tipo de conflicto metodológico, sino que «su esencia sigue las orientaciones fundacionales que nos dejó Kurt Lewin, en su clásico triángulo investigación-acciónformación» (2012: 106).

Las etapas ideales de esta metodología incluirían, por este orden, el diagnóstico del problema, el diseño del plan de acción, su puesta en práctica y posterior análisis, la interpretación de resultados y la posible replanificación (Martínez, 2009: 247-253).

Nuestra adaptación de esta metodología daría lugar a las siguientes fases: 


\section{Fase I. Diagnóstico del problema}

La fase uno, que teóricamente se relaciona con una preocupación o problemática, la hemos denominado «conceptualización del problema».

Sobre esta fase inicial, Antonio Latorre considera que lo más importante es «saber escuchar a otras y otros, saber gestionar la información, saber relacionarse con otras personas, saber implicarlas en la investigación y que colaboren en el proyecto» $(2007: 41)$.

En esta fase, aplicamos diferentes modelos de conversación grupal e individual a dos tipologías de informantes que, a su vez, dividimos en subgrupos:

A. Alumnado:

- A1. Delegados de clase (dos entrevistas semiestructuradas con delegado y subdelegado de cada curso; 14 entrevistas en total).

- A2. Alumnos (recogida de información básica a través de un formulario; $100 \%$ de respuestas).

B. Claustro docente:

- B1. Directores de programa (dos entrevistas en profundidad por director de programa; 12 entrevistas en total).

- B2. Profesores (conversaciones puntuales a través de diferentes medios con profesores con problemáticas particulares; 17 conversaciones documentadas).

Teniendo en cuenta la situación y el punto desde donde partíamos, entendíamos que todos buscábamos encontrar las mejores soluciones para este problema, por lo que la implicación en el proyecto fue alta.

Con los alumnos, en primer lugar, se optó por la vía grupal, dejando que los delegados de cada programa representasen a la totalidad de la clase, ejerciendo de representantes, por lo que podríamos considerar las reuniones mantenidas con cada uno de ellos como entrevistas semiestructuradas a élites (Bourdieu et alii, 1976ः 252-268) que nos proporcionaron su «conocimiento pragmático de la situación» (Santos González y Moreno Carrillo, 2020: 58). Los "ejes de información estratégicos» fueron salud, conectividad y disponibilidad. En segundo lugar, se recogió la información del alumnado general, de forma individual, con el método empírico-analítico (Bisquerra, 2014), a través de formularios para recoger información específica acerca de su situación en relación con los ejes estratégicos mencionados.

Con los docentes, la recogida de información fue mayoritariamente grupal a través de reuniones con los directores de programa, manteniendo también comunicaciones de forma individual a través de correo electrónico, llamadas y videoconferencias con los diferentes docentes de los programas con situaciones 
que debían tratarse de manera individual, pero que proporcionaban información extrapolable al grupo.

Las reacciones de los informantes ante las medidas que la escuela anunciaba como posibilidades también nos proporcionaron información de relevancia.

Fases II y III. Plan de acción, diseño y puesta en práctica

Algunos autores establecen la fase dos como el momento de «coconstrucción de un plan de acción», lo que «implica encuentros con los interesados, a fin de delinear las acciones» (Colmenares, 2012: 107). En este caso nos vimos forzados a conjugar el diagnóstico del problema (fase 1) y el diseño del plan de acción (fase 2 ), ya que para conceptualizar el problema y tomar decisiones de forma inmediata era clave saber de primera mano qué acciones podíamos llevar a cabo en total coordinación con docentes y alumnos.

Con los alumnos se llevó a cabo un grupo de discusión por curso con todos los estudiantes que quisieron participar. La participación media fue del 60\% (95 alumnos de 158 posibles participaron entre los siete grupos de discusión).

Con los profesores se dividió el claustro en dos equipos y se organizaron sendos grupos de discusión. La media de participación fue del $80 \%$ —en total participaron 58 docentes de un total de 72-. Además, en algunos programas se crearon de forma autónoma grupos de trabajo y autoayuda entre profesores.

La puesta en práctica del plan de acción (fase 3) la denominamos «soluciones adoptadas». Dentro del plan de acción se encuentran las soluciones adoptadas tanto para el alumnado como para el profesorado. Las dividimos en plan de comunicación, reuniones y seguimiento y formación.

Fase IV. Reflexión e interpretación de resultados

La última fase la identificamos como el momento de consolidación del informe cuyo objetivo es conocer la eficacia de nuestras acciones. A esta fase la hemos denominado «medición de soluciones», e incluye, además, el registro de las acciones realizadas. En este apartado de medición, se continuaron aplicando técnicas de conversación informal con los alumnos y docentes con mayores problemas para adaptarse al nuevo entorno. Se volvieron a realizar una entrevista semiestructurada a los delegados y subdelegados de los siete programas, y una entrevista semiestructurada a los seis directores de programa, y, además, se distribuyó una encuesta de calidad a la totalidad del alumnado, con un índice de respuesta del 100\%.

En cada una de las fases, diferentes métodos y técnicas de investigación propias de las ciencias sociales han sido aplicados combinando el análisis cuantitativo y cualitativo, si bien con un mayor peso de este último. 
Por tanto, basándonos en los principios de la triangulación de técnicas, en la investigación se han aplicado diferentes herramientas de conversación con diferentes grados de estructura, entrevistas no estructuradas y semiestructuradas, diferentes tipos de paneles y de grupos de discusión, además de encuestas.

\section{El proceso de digitalización a través de la investigación-acción participativa}

Cuando comenzó la pandemia, las instituciones docentes se vieron inmersas en una carrera para intentar continuar con su labor formativa. El confinamiento supuso una situación novedosa y dejó entrever algunas carencias de la digitalización en la educación.

Pese a que nuestro caso particular se enmarca en formación especializada de posgrado universitario, es importante realizar un pequeño análisis macro que nos ayude a comprender de manera general cuál era la coyuntura de la formación, en las diferentes etapas, durante las diferentes fases de la pandemia producida por la covid-19 en España.

El decreto de estado de alarma contenía en su art. 9 una serie de «medidas de contención en el ámbito educativo y de la formación»:

1. Se suspende la actividad educativa presencial en todos los centros y etapas, ciclos, grados, cursos y niveles de enseñanza contemplados en el artículo 3 de la Ley Orgánica 2/2006, de 3 de mayo, de Educación, incluida la enseñanza universitaria, así como cualesquiera otras actividades educativas o de formación impartidas en otros centros públicos o privados.

2. Durante el período de suspensión se mantendrán las actividades educativas a través de las modalidades a distancia y «on line», siempre que resulte posible (RD 463/2020, de 14 de marzo).

Esta decisión, que, tal y como establece la Constitución española, inicialmente duraba 15 días, abría la puerta, como hemos explicado anteriormente, para poder ofrecer durante estos días los programas presenciales de manera telemática sin incurrir en ningún tipo de problema legal.

Ante la naturaleza de las aulas, como lugares cerrados y de concentración de personas en un espacio pequeño, la «virtualidad» pronto dejó de ser temporal (Orden EFP/365/2020, de 22 de abril).

Por tanto, todas las etapas educativas dejaban de ser presenciales. Algo logísticamente bastante factible, especialmente en entornos urbanos, sin entrar, por supuesto, en cuestiones sociales y de acceso efectivo a equipos informáticos e in- 
ternet. Sin embargo, pronto quedó patente que la digitalización de la educación no estaba tan avanzada como se presuponía. Esta situación se convirtió en especialmente grave en las etapas de educación obligatoria, pero era común a todas las etapas educativas, por lo que pronto se convirtió en un tema de actualidad nacional que fue abordado por numerosos análisis periodísticos que recogían las quejas de alumnos y profesores. «La cuarentena que padecen los centros educativos evidencia las deficiencias digitales del sistema, pese a la gran cantidad de recursos disponibles y la buena voluntad de docentes y alumnos», afirmaba en un contundente titular Jorge García en el especial de Retina de El País el 23 de marzo de 2020.

Estas deficiencias son muy variadas; aquí profundizaremos sobre las que nosotros experimentamos. Siguiendo nuestro compromiso con todos los participantes, mantenemos su anonimato, por lo que evitaremos los detalles que pudieran comprometerlo.

\subsection{Fase I. Conceptualización del problema}

En la adaptación de nuestra escuela a la docencia online en el curso 2019/2020 vivimos dos etapas. En la primera tuvimos que encontrar una solución para continuar dando clases durante el «primer» confinamiento.

Puesto que una parte de nuestra escuela universitaria ya estaba centrada en la enseñanza online (aunque dichos programas tienen características y metodologías muy diferentes a la presencial), cuando se planteó el confinamiento decidimos utilizar la plataforma de docencia telemática en los programas presenciales (donde ya se hacía un uso parcial de esta), es decir, incluir aulas virtuales con las que seguir ofreciendo los contenidos previstos. Sin embargo, disponer de las capacidades técnicas no significaba estar preparados para hacerlo tan inmediatamente como pensábamos. Comprobamos rápidamente que la transición a la docencia online no iba a ser tan sencilla como imaginábamos.

\subsubsection{Problemas logisticos y operativos}

En cuanto se confirmó la posibilidad de replicar el modelo online, parte de la logística parecía estar solucionada, ya que alumnos y docentes, simplemente, verían una nueva pestaña en el campus virtual con el que ya trabajaban. Esta nueva sección, denominada «sesiones online», es simplemente el link con el que se integra la herramienta de videoconferencias del campus virtual.

Sin embargo, pronto surgieron otras cuestiones. Alumnos y profesores no estaban preparados para una transición tan brusca al modelo telemático. 
La primera fase de la investigación, desarrollada para abordar este problema, se realizó aplicando diferentes técnicas de conversación y de análisis de las comunicaciones recibidas por parte de equipo académico, claustro y alumnos. Especialmente útiles fueron las conversaciones individuales con representantes de los alumnos y directores de programa, complementadas, más adelante, con conversaciones grupales con los claustros de los diferentes programas.

Los principales problemas recaían, además de en aspectos logísticos, en cuestiones operativas. Como veremos en el apartado siguiente, nos vimos en la necesidad de capacitar a alumnos y docentes en la utilización de la plataforma de streaming, ya que la mayoría de ellos no la había usado antes.

Una vez finalizado el curso escolar 2019/2020 se recuperó la presencialidad en las aulas. Nuestra escuela optó por dar la opción de acudir a la clase presencialmente o seguirla telemáticamente.

Esta solución híbrida supone un mayor número de variables en el aula: cámaras, micrófonos y conexión a internet. Sin olvidar la necesidad de aplicar los protocolos sanitarios de distanciamiento entre estudiantes y el plan y las medidas de actuación ante posibles casos de covid-19 en el aula.

\subsubsection{Problemas metodológicos}

Las clases presenciales de los programas a tiempo completo del curso 2019/2020 se impartían de lunes a viernes en horario de 9 a 14 los programas de mañana y de 16 a 21 en los de tarde. También había programas a tiempo parcial los miércoles y jueves de 17 a 22 .

Aunque alguna asignatura se dividía entre dos docentes, lo habitual es que un profesor tuviese clase cuatro horas y media, excluyendo la media hora de descanso. Mucho tiempo con un mismo grupo, por lo que siempre se esperaban clases prácticas y dinámicas. En general, la metodología aplicada es la de estudios de caso y de resolución de problemas y actividades, además de fomentar la participación del alumnado, si bien siempre hay una presencia significativa de carga teórica y actividades prácticas fuera del aula (Travé-Molero et alii, 2019).

Al trasladar el calendario presencial a online, se evidenció que era mucho más difícil aplicar esta metodología y conectar con el alumnado para que este mantuviese la atención.

En el curso híbrido, descubrimos, a través de nuevas entrevistas a los representantes de los estudiantes y conversaciones informales con alumnos, que el problema metodológico se acrecentaba al tener alumnado presencial, conectado online en directo y alumnos que visualizan las sesiones de manera asíncrona, mientras que la duración de las sesiones se mantuvo. Además, muchos de los 
profesores que debían empezar el curso no habían asistido a las formaciones porque su carga docente se concentra en el primer cuatrimestre y creyeron que no se verían afectados por estos cambios, por lo que nos encontramos con problemas operativos una vez más.

\subsection{Fases II y III. Soluciones adoptadas}

Para solucionar estos problemas se desarrolló un plan de acción y se puso en práctica. El diseño y la implementación corresponden a las fases dos y tres de la metodología.

Como señalamos más arriba, la comunicación constante con alumnos y profesores jugó un papel fundamental en esta fase.

La primera batería de acciones se adoptó desde la coordinación académica tras las primeras reuniones con directores de programa y representantes de alumnos, realizando las siguientes iniciativas, divididas en acciones hacia el profesorado y hacia el alumnado:

Alumnado:

- Plan de comunicación a través del campus virtual: se realizó una comunicación constante de las medidas que se iban adoptando.

- Reuniones virtuales con los delegados y subdelegados de las clases: para recordar las medidas y tener retroalimentación de las necesidades de los diferentes grupos.

- Seguimiento individualizado: a través del correo electrónico y del teléfono para conocer y comprender todas las casuísticas logísticas del alumnado y de los docentes. También para solucionar dudas del campus virtual.

- Píldoras formativas: realización de vídeos de formación sobre las diferentes herramientas del campus virtual.

Docentes:

- Plan de comunicación a través del correo electrónico y del teléfono: información de las medidas y acciones implementadas.

- Píldoras formativas: realización de vídeos de formación sobre las diferentes herramientas del campus virtual.

- Reuniones virtuales con los docentes y los directores de programa: seguimiento de las asignaturas, formación y adaptación de la metodología presencial a la online.

Una vez solucionados los primeros problemas, y ante la evidente falta de dominio del campus virtual y de la herramienta de videoconferencias, se programan diferentes formaciones, una para alumnos y varias para el claustro docente. 
Desde la coordinación académica se programaron tres sesiones formativas en las que se explicaron aspectos básicos del campus virtual y la herramienta de videoconferencias para que aquellos docentes con más problemas pudieran al menos impartir sus clases y evaluar los conocimientos adquiridos.

Además, se programaron sesiones bidireccionales en cada uno de los programas para que los propios docentes compartiesen sus experiencias. Tras estas sesiones de buenas prácticas, cada «descubrimiento» que podía suponer una mejora en la docencia se compartía por correo electrónico.

Esta fase supone un momento de generación de conocimiento en torno a una problemática común. Esto, en algunos casos, cohesionó a un claustro que antes casi ni se conocía personalmente.

Tabla 1. Ejemplo de aportaciones de buenas prácticas para clases telemáticas.

\begin{tabular}{|c|c|c|c|c|c|}
\hline Docente 1 & Docente 2 & Docente 3 & Docente 4 & Docente 5 & Docente 6 \\
\hline $\begin{array}{l}\text { Conectarse } 10 \\
\text { minutos antes de } \\
\text { las clases. }\end{array}$ & $\begin{array}{l}\text { Yo les digo que } \\
\text { preparen una } \\
\text { presentación en } \\
\text { grupo sobre el } \\
\text { tema que trabajar } \\
\text { con presentación } \\
\text { de Google } \\
\text { y que luego } \\
\text { para presentar } \\
\text { comparta uno de } \\
\text { ellos. Hay que } \\
\text { darles permiso de } \\
\text { presentador. }\end{array}$ & $\begin{array}{l}\text { Dividir la } \\
\text { presentación en } \\
\text { varios módulos. }\end{array}$ & $\begin{array}{l}\text { Si es posible, } \\
\text { tener dos } \\
\text { portátiles listos; } \\
\text { asignar el rol de } \\
\text { presentador y } \\
\text { autoenviarnos } \\
\text { el enlace al otro } \\
\text { portátil. }\end{array}$ & $\begin{array}{l}\text { Utilizar Meet } \\
\text { Google como } \\
\text { herramienta } \\
\text { para tener dos } \\
\text { sesiones abiertas. }\end{array}$ & $\begin{array}{l}\text { Marcar las } \\
\text { reglas de clase } \\
\text { e incidir en que } \\
\text { la participación } \\
\text { activa tiene un } \\
\text { peso importante. }\end{array}$ \\
\hline $\begin{array}{l}\text { Verificar que } \\
\text { todos los } \\
\text { alumnos saben } \\
\text { cómo usar las } \\
\text { herramientas, } \\
\text { micro, levantar la } \\
\text { mano, etc. }\end{array}$ & $\begin{array}{l}\text { Yo les pongo } \\
\text { ejercicios cortos } \\
\text { para trabajar, } \\
\text { presentar y } \\
\text { comentar. Los } \\
\text { preparan en } \\
\text { grupos aleatorios. }\end{array}$ & $\begin{array}{l}\text { Utilizar Google } \\
\text { Chrome para } \\
\text { los vídeos, } \\
\text { compartir } \\
\text { pestaña para } \\
\text { ver y escuchar, } \\
\text { cargar antes } \\
\text { los vídeos en el } \\
\text { navegador. }\end{array}$ & $\begin{array}{l}\text { Presentación } \\
\text { atractiva y slides } \\
\text { claros, con poca } \\
\text { información por } \\
\text { diapositiva. }\end{array}$ & $\begin{array}{l}\text { Organizar } \\
\text { las clases por } \\
\text { módulos. }\end{array}$ & $\begin{array}{l}\text { Comunicación } \\
\text { oral clara y } \\
\text { precisa. }\end{array}$ \\
\hline
\end{tabular}




\begin{tabular}{|c|c|c|c|c|c|}
\hline Docente 1 & Docente 2 & Docente 3 & Docente 4 & Docente 5 & Docente 6 \\
\hline $\begin{array}{l}\text { Explicar las } \\
\text { «reglas de la } \\
\text { clase», cómo se } \\
\text { puede participar, } \\
\text { hablar, etc. }\end{array}$ & $\begin{array}{l}\text { Yo creo que } \\
\text { para que no } \\
\text { desconecten, } \\
\text { se trata de } \\
\text { que trabajen } \\
\text { continuamente. } \\
\text { Casos y } \\
\text { ejercicios cortos } \\
\text { continuamente. } \\
\text { Y que tengan que } \\
\text { presentar. }\end{array}$ & $\begin{array}{l}\text { Más pausas pero } \\
\text { más cortas. }\end{array}$ & $\begin{array}{l}\text { Profesor activo } \\
\text { «físicamente» } \\
\text { y mentalmente, } \\
\text { atento a los } \\
\text { alumnos, los } \\
\text { micros... }\end{array}$ & $\begin{array}{l}\text { Clases dinámicas } \\
\text { con participación } \\
\text { de los alumnos. }\end{array}$ & $\begin{array}{l}\text { Apuntar las } \\
\text { palabras clave en } \\
\text { el chat. }\end{array}$ \\
\hline $\begin{array}{l}\text { Explicar los } \\
\text { objetivos } \\
\text { académicos de la } \\
\text { clase. }\end{array}$ & $\begin{array}{l}\text { Cada 10- } \\
15 \text { minutos } \\
\text { cambiamos de } \\
\text { actividad, vídeo, } \\
\text { conversación, } \\
\text { debate... }\end{array}$ & $\begin{array}{l}\text { Marcar pausas } \\
\text { de unos minutos } \\
\text { para dejar } \\
\text { al alumno } \\
\text { preguntar, } \\
\text { asimilar, etc. }\end{array}$ & $\begin{array}{l}\text { Siempre dar } \\
\text { tareas para la } \\
\text { próxima clase } \\
\text { online. }\end{array}$ & $\begin{array}{l}\text { Guardar 5-7 } \\
\text { minutos al final } \\
\text { de la clase para } \\
\text { un wrap-up de } \\
\text { las ideas más } \\
\text { importantes de } \\
\text { la clase. }\end{array}$ & $\begin{array}{l}\text { Siempre el } \\
\text { profesor se tiene } \\
\text { que quedar unos } \\
5-10 \text { minutos } \\
\text { después de las } \\
\text { clases para que } \\
\text { los alumnos } \\
\text { puedan preguntar } \\
\text { algo si lo } \\
\text { necesitan. }\end{array}$ \\
\hline
\end{tabular}

\subsubsection{Soluciones para la docencia bíbrida}

La planificación de la docencia híbrida, para el curso 2020/2021 se puede dividir en las siguientes etapas:

\section{Adaptación logística:}

a) Adecuación de aulas y espacios comunes: esto supone retirar mesas y sillas para adaptarnos a los nuevos aforos.

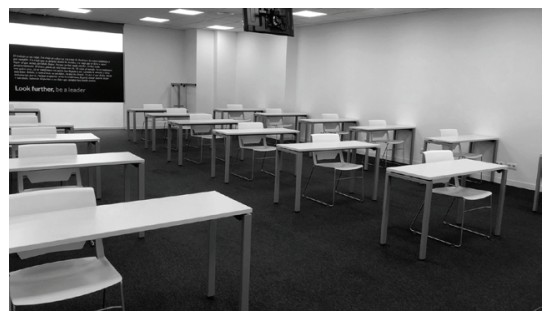

\section{Imagen 1. Aula adaptada a protocolo covid.}

b) La inclusión de un hardware con el que poder comunicar lo que pasa en el aula con el alumnado a distancia. 


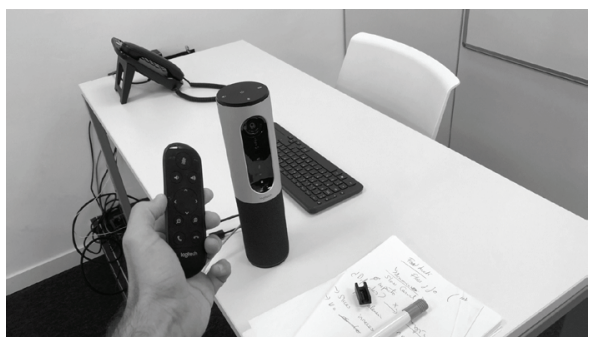

Imagen 2. Hardware para adaptación a la docencia híbrida.

2. Formación para la utilización de los recursos disponibles para la docencia híbrida.

En esta etapa, al igual que en la docencia telemática, se formó a los estudiantes durante los primeros días del curso.

Tras evidenciar numerosos problemas los primeros días de clase, se implementó un calendario formativo, dividido en varias etapas, cuyo objetivo era capacitar a los docentes en el manejo del campus virtual con la mayor soltura y autonomía posible (temporalización octubre-enero), y denominado con el acrónimo $\mathrm{BB}$, y la formación en metodologías para los nuevos entornos digitales.

Figura 1. Calendario de formación 2020/2021.

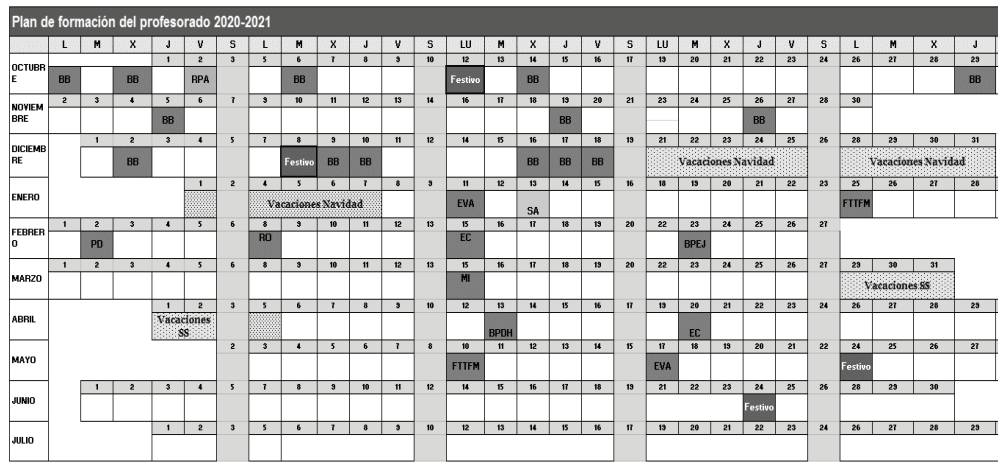


Tabla 2. Leyenda del calendario de formación 2020/2021.

\begin{tabular}{|c|c|c|}
\hline Área & Formación & Código \\
\hline \multirow{4}{*}{$\begin{array}{l}\text { FORMACIÓN } \\
\text { PROFESORADO }\end{array}$} & $\begin{array}{l}\text { Introducción al blackboard y aspectos básicos } \\
\text { para la dinamización en docencia híbrida. }\end{array}$ & $\mathrm{BB}$ \\
\hline & Aspectos básicos de la tutorización del TFM. & FTTFM \\
\hline & $\begin{array}{l}\text { Evaluación en blackboard para profesores y } \\
\text { coordinadores. }\end{array}$ & EVA \\
\hline & Confección y mejora del plan docente. & $\mathrm{PD}$ \\
\hline \multirow{5}{*}{$\begin{array}{l}\text { INNOVACIÓN } \\
\text { DOCENTE }\end{array}$} & $\begin{array}{l}\text { Retos y oportunidades de la metodología } \\
\text { e-learning. Puesta en común. }\end{array}$ & $\mathrm{RO}$ \\
\hline & $\begin{array}{l}\text { Buenas prácticas en la creación de ejercicios } \\
\text { atractivos. }\end{array}$ & BPEJ \\
\hline & Gamificación en entornos virtuales. & MI \\
\hline & Buenas prácticas en dinamización híbrida. & $\mathrm{BPDH}$ \\
\hline & Estudios de caso. & EC \\
\hline \multirow{4}{*}{$\begin{array}{l}\text { FORMACIÓN } \\
\text { INTERNA }\end{array}$} & Seguimiento alumnado. & SA \\
\hline & Cursos reglados formación a distancia. & CR \\
\hline & $\begin{array}{l}\text { Análisis de redes sociales para investigadores } \\
\text { de ciencias sociales. }\end{array}$ & ARRSS \\
\hline & $\begin{array}{l}\text { Estrategias para publicar en revistas de } \\
\text { impacto académico. }\end{array}$ & EPRI \\
\hline
\end{tabular}

Todos estos contenidos quedaron, además, grabados para que su consulta posterior fuera posible.

Además, también se grabaron vídeos explicativos de cada uno de los procesos de manera individualizada.

Figura 2. Documento con links a los vídeos de formación para docentes.

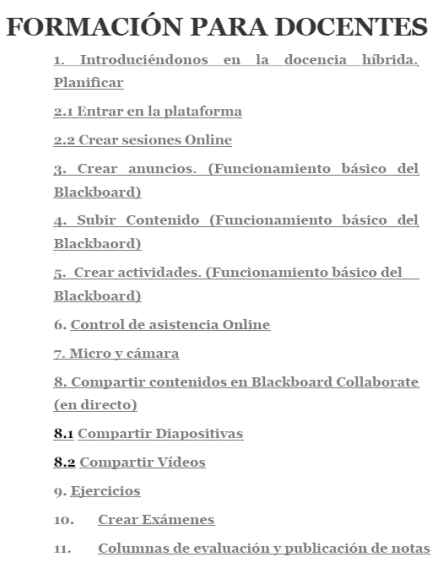




\subsection{Fase IV+ Reflexión e interpretación de los resultados}

Si bien nos estamos ocupando solo de los resultados del campus de Madrid, todo indica que estos son extrapolables a lo ocurrido en el campus de Barcelona; por el momento, solo disponemos de información cualitativa del campus madrileño, por lo que nos centraremos en este caso.

\subsubsection{Resultados cuantitativos}

El análisis de los resultados obtenidos a través de las encuestas de calidad docente refleja el éxito logrado en la adaptación a la docencia telemática durante el segundo cuatrimestre del curso 2019/2020. La comparación de los resultados generales obtenidos por las asignaturas del segundo cuatrimestre en los cursos 2019/2020 y 2018/2019 arroja, de hecho, un resultado positivo para la docencia en línea, 8,18 puntos de media sobre 10 frente a la docencia presencial del curso 2018/2019, 7,74 de media sobre 10 (ver figura 3).

Sin embargo, los másteres en Organización de Eventos y Turismo de Negocios (MICE), tanto en su versión a tiempo completo (en inglés) como en la de tiempo parcial, suponen sendas excepciones (ver figura 3). Algo parecido ocurre con el máster en Organización de Eventos y Turismo de Negocios a tiempo completo impartido en lengua castellana, que se activó durante el curso 2019/2020 por primera vez, cuya nota media para el segundo cuatrimestre se queda en un 7,93 , ligeramente por debajo del promedio general y muy cerca de los resultados de los otros dos másteres con la misma temática, pero diferentes modalidades (ver figura 4).

Figura 3. Comparación calidad docente del $2 .^{\circ}$ cuatrimestre de los cursos $2018 / 2019$ y $2019 / 2020$.

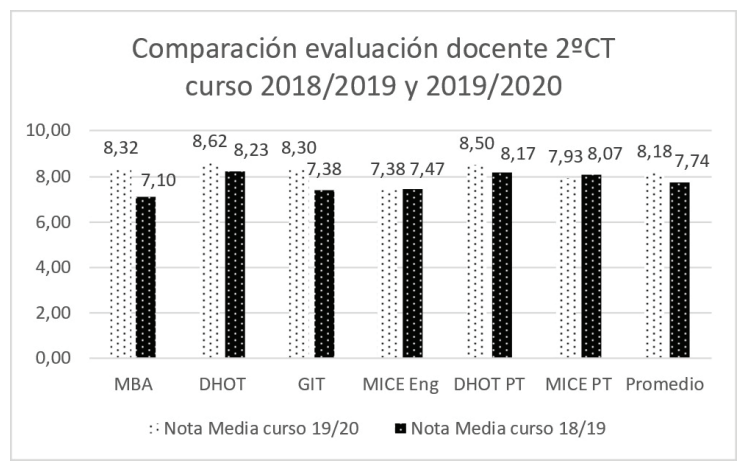

Fuente: elaboración propia. 
Figura 4. Comparación calidad docente del $2 .^{\circ}$ cuatrimestre del promedio general y del máster MICE cast. del curso 2019/2020.

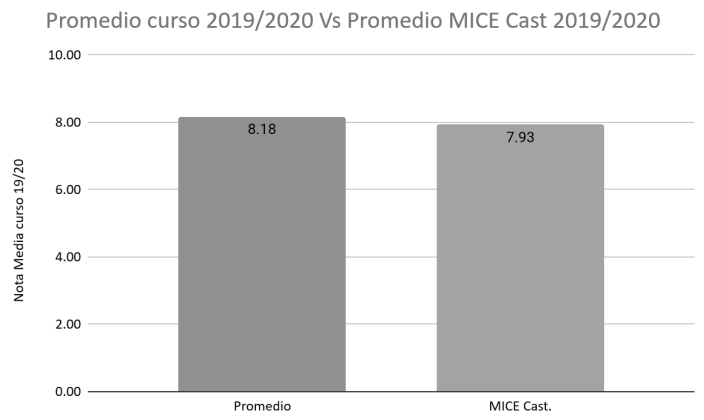

Fuente: elaboración propia.

Teniendo en cuenta que, partiendo de la clasificación por niveles de Harmon y Jones (1999: 28-29), nos encontrábamos entre los niveles dos y tres - dependiendo de la asignatura, parte de los materiales o casi su totalidad estaban disponibles en línea- y nuestro objetivo era acercarnos lo máximo posible al ideal del nivel cinco - crear una comunidad digital de aprendizaje constructivista-, podemos afirmar que cuantitativamente el proceso ha sido relativamente exitoso.

\subsubsection{Análisis cualitativo}

En primer lugar, debemos destacar que la ligera mejora en los promedios de cada máster —en los casos de $\mathrm{MBA}^{2}$ y $\mathrm{GIT}^{3}$, de más de un punto- no parece significar tanto que la docencia telemática haya sido mejor valorada que la presencial como que los estudiantes han comprendido y premiado el esfuerzo de sus profesores adaptándose rápidamente a la nueva situación, tal y como recogen las entrevistas a los representantes de los estudiantes y a los directores de programa. Sin embargo, estos resultados sí demuestran que en general se ha conseguido un uso bastante aceptable de las herramientas telemáticas y que la adaptación metodológica ha permitido una buena transmisión e interiorización de los conocimientos.

Por los mismos motivos, los resultados de los másteres MICE merecen ser analizados con detalle. Si, en general, la evaluación de los estudiantes ha sido generosa y en cursos anteriores sus resultados estaban muy cerca de la media e incluso por encima de esta, por qué en esta ocasión se produce una triple coincidencia en la relativamente baja valoración del estudiantado.

2 MBA en hospitalidad y turismo.

3 Gestión internacional del turismo. 
El análisis cualitativo de las entrevistas realizadas y de los comentarios libres en las encuestas de calidad señala claramente en una misma dirección, se trata de unos cursos y unas asignaturas de enorme carga práctica y profesional cuyas expectativas en este sentido no han sido totalmente cubiertas. Falta de dinamismo, peticiones de más actividades prácticas e interactivas durante las clases, demasiado peso de la teoría frente a la práctica, etc., son quejas y sugerencias ampliamente repetidas. Del mismo modo, los profesores con mejores resultados fueron aquellos que desde el principio aplicaron una metodología más participativa, en estos casos se valoró muy positivamente la utilización de algunas de las herramientas que incorpora el propio sistema de videoconferencias y la plataforma virtual, como encuestas, foros, blogs, wikis, etc., así como de otras que permiten reforzar los conocimientos teóricos de forma lúdica como Kahoot ${ }^{4}$, y muy especialmente de los casos prácticos resueltos de manera colaborativa, bien por toda la clase o bien por subgrupos creados con el sistema de videoconferencias.

El análisis cuantitativo y cualitativo, asignatura a asignatura y profesor a profesor, corrobora esta impresión y la acentúa respecto a cursos anteriores. A mayor peso de la clase magistral, peor valoración, y a mayor utilización de actividades prácticas y colaborativas, mejores notas.

Una de las consecuencias que hemos detectado ante clases con mucha carga teórica y una metodología menos activa es que en ocasiones algunos de los alumnos telemáticos síncronos pierden el hilo de la explicación y prefieren desconectar a interrumpir al profesor. A estos alumnos los llamamos «des-conectados», permanecen formalmente en la sesión pero dejan de prestar atención a los contenidos y a la dinámica de la clase.

Estos resultados corroboran, como apuntaba Adell hace más de quince años, que para algunos profesores la docencia telemática fue entendida como una mera transposición de la docencia presencial a un entorno digital (2004). Del mismo modo, la creación o adaptación al ámbito digital de materiales y sistemas de evaluación resulta imprescindible para obtener resultados satisfactorios (Jolliffe, Ritter y Stevens, 2001; Garrison y Anderson, 2005; Pallof y Pratt, 2001). En este sentido, aquellos docentes que han ejercido en mayor medida siguiendo el modelo de mediador digital de Salmon (2000) han obtenido mejores resultados de satisfacción por parte de los estudiantes.

Podemos afirmar que estas tendencias están presentes en todos los cursos y materias, pero que se acentúan claramente cuanto mayor es la expectativa de interactividad por parte de los estudiantes.

4 Aplicación informática que usando los teléfonos inteligentes permite diseñar cuestionarios en forma de competición entre estudiantes o grupos de estudiantes. 


\subsubsection{El papel del claustro y de los directores de programa}

Esto nos sitúa ante el hecho de que nuestra formación a los docentes, a la que asistieron virtualmente o vieron grabada alrededor del $80 \%$ del profesorado y prácticamente el $100 \%$ de quienes impartían clase durante el segundo cuatrimestre, se centró en un primer momento en la capacitación técnica dejando en meras recomendaciones y alusiones a algunas herramientas (como las encuestas o los subgrupos que ofrece la herramienta de videoconferencias) la formación metodológica.

En este sentido se expresaron todos los directores y directoras de los diferentes másteres tras recoger las impresiones de su claustro. La urgencia de la adaptación y las importantes lagunas técnicas que detectamos hicieron que minusvalorásemos la necesidad de capacitar metodológicamente a un profesorado acostumbrado a la interacción en el aula, pero no a la interacción digital. Si bien la comunicación constante con docentes y estudiantes nos permitió ir detectando estas carencias, nuestra capacidad de intervención fue muy limitada.

Debemos destacar que los mejores resultados promedio se dan en los másteres en Dirección Hotelera y Restauración (DHOT): 8,62 y 8,50 puntos. Una de las razones que explica este éxito se encuentra en la implicación del director de ambos programas en la formación metodológica de su claustro. A las pocas semanas de comenzar con la docencia online, por iniciativa propia, convocó a todos los profesores de los dos másteres para que quienes ya habían dado clases compartiesen sus experiencias y entre todos construir una lista de buenas prácticas y consejos metodológicos que han hecho que los comentarios negativos de los estudiantes sean sensiblemente menos frecuentes que en el resto de los cursos. Esta formación entre iguales se repitió en el resto de los másteres, pero la implicación conseguida fue sensiblemente menor.

Teniendo esto en cuenta, consideramos normal que un buen número de estudiantes haya coincidido en que las clases más teóricas «se hacían muy largas», que «resultaba muy difícil mantener la atención frente a la pantalla durante las explicaciones» o, directamente, «eran muy aburridas, prefería hacer otra cosa y repasar por mi cuenta los materiales».

\subsection{Resultados de la docencia híbrida}

La detección de estos problemas nos ha permitido afrontar mejor preparados (o al menos eso creemos) el comienzo del curso 2020/2021 en el que la docencia comenzó (continúa cuando escribimos este artículo a comienzos de 2021) bajo el modelo que hemos denominado híbrido. 
Aunque todavía no disponemos de datos cuantitativos sobre el nivel de satisfacción del alumnado, las entrevistas a los representantes de los estudiantes y la comunicación constante con el claustro docente sí nos permiten apuntar algunas de las tendencias detectadas.

De manera generalizada el profesorado ha interiorizado que se dirige a tres tipos de estudiantes, los presenciales, los telemáticos síncronos y aquellos que ven las clases en diferido. Esto implica seguir usando las herramientas que proporciona la plataforma de videoconferencias, estar atento a la cámara que hay en el aula y al plano que ofrece a los estudiantes no presenciales, así como sustituir la pizarra de clase por la virtual de la plataforma, con sus ventajas e inconvenientes. Pero, sobre todo, exige un gran esfuerzo para implicar y atender con la mayor equidad posible a los tres colectivos de estudiantes. Conscientes de esto, hemos animado al claustro a utilizar metodologías prácticas y participativas, se ha formado en el uso de casos prácticos, se han compartido experiencias con herramientas de ludificación en el aula para facilitar la impartición de contenidos teóricos y se ha recordado que deben ofrecerse actividades alternativas a los alumnos asíncronos cuando sea necesario.

El resultado de estos esfuerzos parece estar teniendo una respuesta positiva por parte tanto de los estudiantes presenciales como de los que se conectan de forma sincrónica, si bien estos últimos se han podido sentir algo olvidados en ciertos momentos debido a la falta de atención de algunos profesores a la posición de la cámara o al uso de la pizarra del aula, con mala visibilidad para este colectivo.

Los mayores problemas los estamos detectando entre el estudiantado que ve las clases en diferido, en su caso, cuanto más participativas son las clases, más alejados de estas se sienten y menos dicen aprovecharlas. Para este grupo de estudiantes hemos promovido la utilización de herramientas que siguen «vivas» fuera del horario de clase, como foros, blogs o wikis. Sin embargo, a pesar de las actividades complementarias sigue habiendo muchos momentos en los que ven participar a sus compañeros sin posibilidad de hacerlo ellos ni de obtener una respuesta directa por parte del profesor. Fundamentalmente, nos encontramos ante un problema de construcción de ecosistemas tecnológicos (García-Peñalvo, 2018) y comunidades digitales de aprendizaje (Palloff y Pratt, 1999; Cabero, 2006). Los estudiantes asíncronos quedan casi totalmente fuera de la comunidad mixta — digital/presencial—, que sí se ha conseguido forjar entre los estudiantes presenciales y en línea. Estos estudiantes nos han señalado, por tanto, la sensación de soledad y aislamiento que experimentan, las dificultades para seguir los itinerarios formativos de cada asignatura y la desmotivación que ha llevado 
a algunos de ellos incluso a abandonar el curso. Esta contradicción entre lo que funciona para los estudiantes presenciales y en línea y lo que funciona con los alumnos asíncronos nos vuelve a situar ante la necesidad de repensar la metodología y la organización de las clases.

\section{Conclusiones}

Aproximadamente un año después de iniciar el proceso de digitalización de esta escuela universitaria, con la transformación de la docencia presencial a los formatos telemático e híbrido, podemos afirmar que la clave del éxito no ha sido la posibilidad logística de implementar aulas virtuales que emulasen las presenciales, sino que ha residido en la continua mejora de los procesos, principalmente logísticos, pero también metodológicos dentro de la organización.

Para esta adaptación constante y mejora en procesos, ha sido clave formar tanto a los profesionales encargados de impartir nuestros servicios (docentes) como a nuestros clientes (alumnado), sin olvidar que debíamos comunicar este éxito al cliente prospectivo.

Inicialmente las formaciones fueron de carácter general y fundamentalmente técnico, enfocadas en la utilización de la plataforma de docencia virtual y especialmente de la herramienta de videoconferencias, asegurándonos de la autonomía del profesorado a la hora de grabar las sesiones y de su capacidad para resolver pequeños problemas de imagen y sonido.

Con el objetivo de mejorar la satisfacción de nuestros clientes, nos vimos en la necesidad de ofrecer una formación enfocada a la utilización de nuestra plataforma online, especialmente en el curso 2020/2021. Con la progresiva vuelta a las aulas, implementamos el formato de docencia híbrida y nos vimos en la necesidad de ofrecer una formación más transversal, ya que no solo necesitábamos una adaptación operativa, sino que también era capital una adaptación metodológica en el uso de herramientas (algunas de la propia plataforma virtual y otras de la docencia más innovadora presencial) para ofrecer los contenidos docentes de una forma más dinámica y participativa.

Nuestro objetivo principal ha sido en todo momento que los resultados formativos de nuestros estudiantes no se vieran afectados. Por ello una de las primeras decisiones adoptadas fue no reducir los temarios ni la carga docente y modificar lo menos posible los criterios de evaluación, a pesar de que desde el principio detectamos ciertas dificultades, tanto entre alumnos como entre profesores para permanecer concentrados durante clases de hasta dos horas y cuarto seguidas frente a la pantalla del ordenador. 
Incluso tras la formación metodológica del claustro nos encontramos ante el problema de la transmisión de conocimientos teóricos, mucho más difíciles de adaptar a las modalidades telemática e híbrida, pero imprescindibles en la formación universitaria de calidad.

Nuestra intención ha sido que estos conocimientos teóricos pudieran transmitirse del modo más participativo posible, intentado reducir las clases magistrales - con peor funcionamiento fuera de la presencialidad del aula - y primar la utilización de ejercicios prácticos y enseñanza entre iguales. No obstante, todavía queda camino por recorrer para que todos los profesores encuentren la forma más adecuada de adaptar su materia a esta nueva realidad.

En este sentido, es imprescindible dar continuidad a la formación y hacer consciente al profesorado de la necesidad de estar preparados para la docencia telemática e híbrida, pues esta no va a desaparecer con la covid-19 sino que va a seguir usándose (con intensidades diferentes) en el futuro. Por tanto, es imprescindible mantener una formación continua en herramientas técnicas y en metodologías activas para evitar, ante una necesidad parecida, la baja capacitación digital que encontramos en la transición a la docencia telemática.

Debemos volver a destacar la importancia de la adaptación metodológica y la necesidad de establecer un seguimiento continuo de la actitud de los estudiantes para evitar la desconexión de estos cuando se encuentran en línea, síncrona o asíncrona. Para ello, la participación continua y el aprendizaje colaborativo se han mostrado como las técnicas con mayor éxito.

Nos planteamos si es posible implementar con éxito un modelo docente capaz de cubrir las necesidades y los objetivos de aprendizaje de las tres modalidades diferentes de alumnado a las que nos hemos enfrentado.

Todo indica que es posible compaginar la atención al alumnado presencial y telemático síncrono si se dominan las herramientas digitales necesarias y se adapta la metodología primando la participación de los estudiantes. Sin embargo, parece que nos encontramos ante una contradicción difícil de salvar en lo que respecta a los alumnos telemáticos asíncronos, pues, como apuntábamos más arriba, a mayor participación de los dos primeros grupos, menor sensación de integración y aprovechamiento de la docencia de los segundos, que quedan aislados de la comunidad digital de aprendizaje (Palloff y Pratt, 1999; Cubero, 2006; García Peñalvo, 2018). Esta carencia quizá pueda solventarse dando más peso a las herramientas que dan continuidad a la formación fuera del tiempo de clase (foros, blogs, wikis, etc.), adaptando las actividades de clase o incluso editando las grabaciones. Encontrar una solución satisfactoria para los tres grupos es un reto al que tendremos que seguir enfrentándonos. 
Finalmente, debemos preguntarnos en qué medida las soluciones aportadas durante el confinamiento han podido ayudar a la posibilidad de mantener los programas presenciales (híbridos) en el curso que recientemente acaba de comenzar. El simple hecho de que podamos seguir ofreciendo programas especializados en un sector tan azotado como el turismo, en un momento con tantas limitaciones de movimiento y teniendo alumnos presenciales provenientes de prácticamente todos los continentes, nos hace pensar que hemos aportado soluciones que ayudan a la viabilidad de la escuela en el corto y medio plazo.

\section{Referencias bibliográficas}

Adell, J. (2004). «Nuevas tecnologías en la formación presencial: del curso online a las comunidades de aprendizaje». Qurriculum: Revista de Teoría, Investigación y Práctica Educativa, 17, 57-92.

Azcorra, A.; Bernardos, C. J.; Gallego, O. y Soto, I. (2001). Informe sobre el estado de la teleeducación en España. [Informe en línea]. Universidad Carlos III. Disponible en: <http://www.it.uc3m.es/azcorra/papers/2001_Informe_de_la_AUI_sobre_el_estado_de_la_teleeducacion_en_Espana.pdf $>$.

Bourdieu, P.; Chamboredon, J. Cl. y Passeron, J. Cl. (1976). El oficio del sociólogo. Buenos Aires: Siglo XXI.

Bisquerra, R. (2014). Metodología de la investigación educativa. Madrid: La Muralla.

Cabero, J. (2006). «Bases pedagógicas del e-learning». RUSC. Revista de Universidad y Sociedad del Conocimiento, 3 (1).

Colmenares, A. M. (2012). «Investigación-acción participativa: una metodología integradora del conocimiento y la acción». Voces y silencios: Revista Latinoamericana de Educación, 3 (1), 102-115.

Elliot, J. (1990). A framework for self-evaluation in schools. Cape Town: Univ. of Cape Town.

Freire, P. (1974). Pedagogía del oprimido. México: Siglo XXI.

García, J. (2020) «La odisea de la formación online impuesta por el coronavirus». Retina. El País. Disponible en: <https://retina.elpais.com/retina/2020/03/22/innovacion/1584868868_589467.html>

García-Peñalvo, F. J. (2018). «Ecosistemas tecnológicos universitarios». En J. Gómez (ed.). UNIVERSITIC 2017. Análisis de las TIC en las Universidades Españolas (pp. 164-170). Madrid, España: CRUE.

Garrison, D. R. y Anderson, T. (2005). El e-learningen el siglo Xxi. Barcelona: Octaedro. 
Harmon, W. y Jones, M. G. (1999). «The five levels of web use in education: Factors to consider in planning online course». Educational Technology, 39 (6), 28-32.

Jolliffe, A.; Ritter, J. y Stevens, D. (2001). The online learning handbook. London: Kogan Page.

Latorre, A. (2007). La investigación-acción. Conocer y cambiar la práctica educativa. Barcelona: Grao.

Martínez, M. (2009). Ciencia y arte en la metodología cualitativa. México: Trillas.

Palloff, R. M.y Pratt, K. (1999). Building Learning Communities in Cyberspace: Effective Strategies for the Online Classroom. New York: Jossey-Bass.

Pallof, R. M. y Pratt, K. (2003). The virtual student. San Franciscoः Jossey Bass Wiley.

Prawat, R. S. (1992). "Teachers' Beliefs about Teaching and Learning: A Constructivist Perspective». American Journal of Education, 100 (3), 354-395. Disponible en: <https://doi.org/10.1086/444021>

Salmon, G. (2000). E-moderating. The key to teaching and learning on-line. London: Kogan Page.

Santos González, D. y Moreno Carrillo, J. M. (2020). «¿Es necesario regular la práctica de surf durante el verano?» Barataria, 28, 105-107. Disponible en: <https://doi.org/10.20932/barataria.v0i28.560>.

Schell, G. (2001). «Student perceptions of web-based course quality and benefit». Education and InformationTechnologies, 6 (2), 95-104.

Sung, Y.-T.; Chang, K.-E. y Liu, T.-C. (2016). «The effects of integrating mobile devices with teaching and learning on students' learning performance: A meta-analysis and research synthesis». Computers E Education, 94, 252-275. Disponible en: <https://doi.org/10.1016/J.COMPEDU.2015.11.008>.

TABa, H. y Noel, E* (1957). Action research: a case study. Washington: ASCD

Travé-Molero, R.; Martínez Iglesias, M.; Díaz Rodríguez, P. y Soro, E. (2019). «Observar, Participar, Entender: la Etnografía como Herramienta de Innovación Docente en los Estudios Superiores de Turismo». En F. J. Murillo y C. Martínez-Garrido (coords). Investigación comprometida para la trasformación social, vol. 2 (pp. 345-350). Madrid: AIDIPE. 